\title{
Large Project Management in the Automotive Industry: A Flexible and Knowledge Based Approach
}

\author{
Filipe Ferreira $^{1}$, Ana Luísa Marques ${ }^{1}$, José Faria ${ }^{1,2}$, Américo Azevedo ${ }^{1,2}$ \\ ${ }^{1}$ CESE / INESC TEC, Campus da FEUP, Porto 4200-465, Portugal \\ ${ }^{2}$ Faculdade de Engenharia, Universidade do Porto, Rua Dr. Roberto Frias, Porto 4200-465, Portugal
}

filipe.d.ferreira@inesctec.pt, ana.1.marques@inesctec.pt,jfaria@fe.up.pt, ala@fe.up.pt

\begin{abstract}
This paper presents a novel approach on flexible and knowledge intensive process management, driven by a large automotive industry case study. The automotive company in analysis requires a very dynamic behaviour, based on high flexibility of both people and equipment. Market has been imposing a decreasing of automotive products life cycles, increasing the number of line adaptations during the entire value chain, resulting in an increased complexity from product design to production. To handle this complexity, new knowledgebased methods and technologies to model, simulate, optimize and monitor planned and existing manufacturing systems are required. Existing large Enterprise Information Systems impose totally structured and predictable workflow, while knowledge intensive processes are flexible and unpredictable, involving high amount of human-decision and interaction. This lead to the need of development of highly specialized applications. This paper presents a novel hybrid approach, including work, information and communication management, to support knowledge intensive processes. The application of the new solution in the automotive engineering process management proved to be very effective and efficient, leading to significant savings.
\end{abstract}

Keywords: Hybrid Process Management; Knowledge Based Engineering; Collaborative Engineering; Product Design and Development; Manufacturing Planning.

\section{Introduction}

Nowadays, automotive industry is driven by mass-customization and high quality and productivity standards. Time to market is critical due to the imposed shorter life cycles of the products, increasing the number of facelifts and respective production line adaptations during the entire value chain. This implies a fast reaction and proactive production system based on product, process and human resources flexibility [1] [2].

The presented use case is based on a flexible multi product assembly line that produces three car models. This paper is focused on the planning stamping processes, which involve high levels of collaboration, communication and human decisions. These kind of processes require a very forceful and dynamic behaviour, which is achieved through qualified and motivated people, continuous improvement and innovation.

The company has a specific Business Unity (BU), dedicated to dies construction, together with other four tool shops, supports die constructions as a core technical 
business worldwide. With a workforce around 230 employees, equipped with modern milling machines and try-out presses, this BU is able to follow-up several die-sets projects from analysis phase till the process validation phase.

Every time a new car model is launched, a new die-set project is raised for stamping, been managed by a dedicated team that has to handle a huge amount of information flowing through the different stakeholders and during several phases of project. Having the right information at the right moment, to make fast and well based decisions is usually a challenge planners and managers have to face.

After analysing the as-is processes, it was possible to assert that the continuous updates on each die-set (DS) status were consuming a considerable amount of time and resources, turning the decision making process more reactive instead of the desired proactive approach. It was also possible to see that valuable information is spread by numerous departments and specialists. This approach represents a severe concern since it can be the cause of wrong or slow decisions due to misinformation in a process that is very time dependent.

This show us that this process can possibly generate high amounts of information that need to be searched, organized, reported, kept, tracked and stored for future projects causing the concerns regarding the reaction times and reliability of information.

The current state of the process weaknesses can be summarized and identified in the following list:

- Transversal and hierarchical projects involving several departments and objectives within the organization;

- Human intensive processes with high level of improvement and feedback loops;

- Processes dependent on individual expertise;

- The number of people involved and information's storage formats are incompatible with a pro-active approach;

- Management based on individual trust;

- Low capacity for knowledge capture and reuse;

- Lack of standardized knowledge based databases fed by past experience and personnel knowledge;

- The current storage method for data makes it difficult to gain access to it;

- Ad-hoc communication flow;

- The reliability of the data is compromised because of the communication methods and data formats;

- Time dependent processes that do not allow on-time/online monitoring;

- High risk of misinformation.

Having analysed the as-is state in detail by considering the process mapping it is possible to understand that all the weaknesses can be eliminated by providing means to have a proactive approach in the die-set manufacturing process.

This paper presents a complete solution to support collaboration in knowledge intensive work environments in the scope of automotive engineering and planning, including integrated work, information and communication management. 


\section{Approaches and state of the art}

Knowledge intensive processes are not suitable for traditional automation and enterprise information systems and new knowledge-based methods, technologies and tools are required [1].

It is observed that in many cases the existing information and communication technologies tools are not capable to manage the daily activities due to the lack of flexibility regarding dynamic and changeable environments [3] [4]. Large enterprise information systems impose structured and predictable workflow, while projects are flexible and unpredictable, involving high amount of human-decision and interaction [3] [5]. Moreover, many times, large enterprise information systems are not able to manage the communication and information inherent to the knowledge-based process itself [4] [6]. Thus, during the project execution, team members are forced to use office productivity tools such as spreadsheets and text editors as well as shared folders and internal email and phone to manage processes in flexible and unpredictable work environments [3].

This lead to the need of development of small and highly specialized applications with few features, available in any alternatives (Tablets, Phones, PC) and cross linked in order to support the product designers, process planners, and shop floor managers in their daily activities with a special focus on supporting collaboration in knowledge intensive work environments [3].

Process management research tends to focus on situations that are responsive to formal analysis and modelling. However, in several domains, it is not possible to structure or model processes using formal notations. The total flow of so called semistructured processes is not known a priori and many times, only the high level phases are defined [1] [8].

The characteristics of a structured process include:

- The scope is totally defined and all the activities are known;

- It is possible to identify the initial state, the final state, and all potential transitions;

- The process is suitable for fully automation.

Despite the apparent simplicity of the above characteristics, many companies try to completely automate this kind of processes with no success due to the fact that a full definition of a knowledge based process is not possible [6] [8] [9].

In fact, humans are better than computers for non-structured and ambiguous situations [1]. On the other hand, computers tend to be better than humans for full structured processes. This situation lead to a more difficult decisions where companies need to determine when a process should be fully automated or not [10] [11].

Some important implications of non-structured processes include [10] [13]:

- Inputs may not be completely definable;

- A priori optimization is not definable since optimization implies the use of rules;

- Increased flexibility, since a non-structured process cannot be fully described.

The case presented in this paper falls into these two extremes and when a new car project starts, the top management defines only some milestones that are controlled in an automatic way. However, the work to be done between each milestone cannot be 
automated once it relies on human intensive knowledge and decision tasks, even if the final results are known. The combination of these structured and unstructured activities are equally important in terms of leading the project to its success [10].

In summary, decision making involve two types of processes: a structured process is a process that can be reduced to fully-defined rules leading to high efficiency but low flexibility (typical workflow management systems), while a non-structured process is not reducible to fully defined rules, leading to high flexibility but low efficiency and control (typical ad-hoc managed processes). We call a process as semi-structured when it contains both structured and non-structured sub-processes leading to a flexible and efficient hybrid approach. This kind of processes are suitable to be supported for what we call Hybrid Management Systems, as depicted in Fig 1.

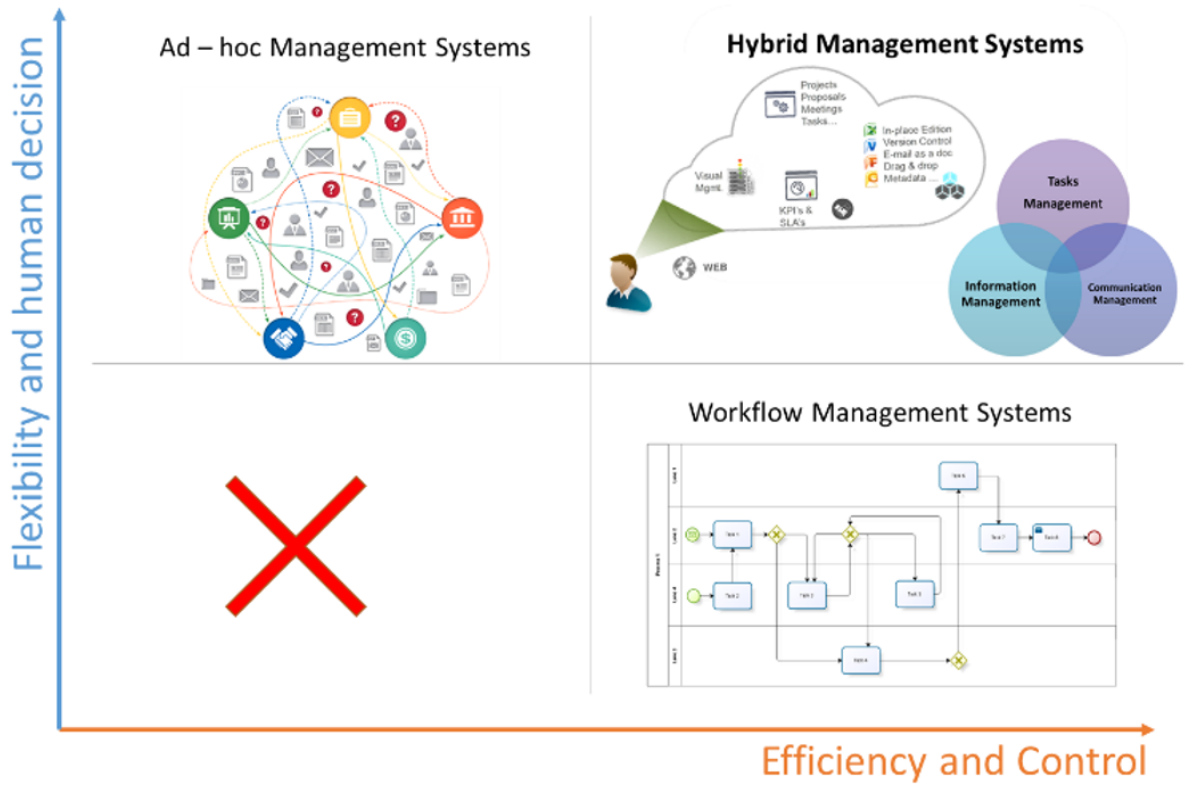

Fig. 1. Flexibility vs. Efficiency in Process Management

\section{Detail analysis of the process}

The die-set development process was split into three main stages: planning, machining and evaluation stages. In fact, each process instance can be deployed and implemented in partnership between the buyer and suppliers, or simply by the buyer or the supplier. Here, the decoupling point depends on the level of expertise of the buyer to deploy and control this process.

The planning is one of the most critical stage of the DS development process, since the outcomes of the activities involved can strictly affect the performance of the overall process execution. Based on the assumptions that the DS specifications have, the 
following activities must be performed: Team Definition, Simulation \& Feasibility Study, Capacity \& Resources Allocation, Die Concept \& Process Simulation and Components Design.

Starting from the team definition, each technician is responsible for a series of diesets, distributed to a small number of suppliers (one or two maximum in order to enhance the buyer-suppliers relationship). Then it is necessary to execute the right simulations and feasibility tests, in order to understand if the technical specifications of each die-set are correct from a product and manufacturing point of view. After validation of the technical specification for each die-set, it is important to understand if the plant, where the die-set is going to be used, has the resources necessary to apply the die-sets designed. In other words, if the facilities have enough space to store the diesets, during the "homeline" try out and production, as well as understand if there is any available press capable to apply, for instance, the forces and torque necessary. Known and validated the die-sets, as well as the necessary resources, it is possible to build a 3D model of each die-set, as well as plan the die-set construction, in terms of budget, time and quality. Here it becomes critical to specify the quality standards to be validated at the end of the process execution.

The following stage, the machining, is where the supplier has to transform the dieset 3D model into a final and usable die-set, capable to produce the parts as specified by the product owner.

The final stage must be focused on the evaluation and improvement of the die-set model built, towards the successful production of the idealized parts. Here, both company requirements and quality standards defined during the planning stage must be taken into account. Thus, as first action, technicians must build some prototype parts in order to validate if the dies are fulfilling the expected quality standards. After all product requirements being validated the manufacturing process is tested thought a preseries evaluation. If minor problems are identified, then correction action must be taken in buyer's house, following an iterative approach.

Given this scenario, it is clear that managing a die-set program for a new car project or for a new car variant requires a holistic view of managing several hundreds of individual instances that depend on each other. When some decisions are being made individually it can affect other running projects. For example a decision in the development of one DS can have indirect consequences in other running DS development projects. Additionally, it requires managing several suppliers, stakeholders and documents that are being continuously updated into new versions due to improvements or unpredictable changes in the product development. The challenge is how to manage effectively the product development process when the information collected is dynamic, comes from multiple sources (stakeholders and software tools) and has dependences.

The process of dies manufacturing is highly complex and very human intensive (only highly skilled resources based on experience and expertise are chosen). It has a step-by-step methodology with a high level of improvements and feedback loops, through the lifecycle of the product development, demanding a very flexible and real time monitoring process. 
Despite this, the complexity of the DS development process increases because each step can be performed by a different expert team with different goals. The existing interdependencies must be taken into account to avoid lack of consistency. Additionally, there is a huge amount of information and communication flow being managed.

The need to control these flexibility due to product development time constrains led to the development of a Hybrid Process Manager (HPM) application that is helping to control the several steps and sub-steps, tasks and activities of the process in terms of time, information and communication flow. Moreover, information generated along the different activities of the process life cycle, is classified and catalogued according to its relevance for the process execution, in order to optimize the time and effort necessary to make decisions.

The product development communication process was being made through the traditional support form of paper or e-mail, which was easily forgotten, lost or ignored when the work environment have excess of information. The developed communication functionalities allow connecting all stakeholders and interdisciplinary teams that are involved in the product development. This guarantee real-time communication, information regarding updated documents, problems or other issues, saving time and improving the decision making process.

As stated before, the product development process of building a new die, requires a lot of expertise, experience and knowledge that are valuable on solving problems and which were not captured, stored or re-used. The knowledge reuse process will be made through ticketing functionalities where the user can document in detail the problem of the part being developed, list of actions, method, solution, and verification and action plan. This information can be re-used when needed through a database with a problemsolution-result repository.

The product development process, as stated before, is very complex and requires the management of a high quantity of tasks with their problems and constrains. In order to keep track on the status of the tasks it will be monitored by an application which will allow the user to identify if a task has a delay.

Traditional process management is mostly mechanical, mono-causal and nondynamic with a linear structure that is unsuitable to solve the above challenges. The flexibility desired due to the dynamic system of product development requires a new approach that will allow to breakdown the communication and collaboration barriers between the product-process-factory-production levels. The adoption of the developed tool supports the company in facing this challenge and enables a faster and a more efficient product development process.

\section{Solution}

The aim of the Hybrid Process Manager solution is to facilitate the flow of information among partners, making it more reliable and actual, allowing a closer control and fast reaction. Thus, it is strictly linked with planning activities on the stamping area, covering a special challenge related with the launch of a new model. 
Working in cooperation with other departments or external partners, stamping planning department has to manage a pack of dies-sets (the tooling necessary to produce new parts for a new model), track the flow path, schedule, define milestones, handle project changes, evaluate risks and define actions, as well as control costs and capacities along the project development.

In order to successfully accomplish this mission, for each project this department need to collect and manage huge amount of information and knowledge, coming from inside and outside of the factory. Indeed, managing all this information not only is intensively time consuming, slowing down decision-making process, but also hinders project reliability, increasing its uncertainty.

Specifically, the aim of the solution is to manage the product development process. Currently these actions have been done manually using numerous physical, electronic files and calls. Therefore all the information needed and used in the process is spread around several departments, reducing the reliability of the entire process to a minimum. To solve this, a new approach and solution is needed.

HPM solution addresses the main challenges that are typical in this kind of semistructured and knowledge intensive collaborative environments by implementing the following functionalities:

- Dashboard for holistic view of project status;

- Real time monitoring and control of the ongoing work activities;

- Totally integrated knowledge and documents management;

- Technical integration with Windows and Office tools, which are widely used by project teams;

- Integrated communication/messaging along the process activities in the scope of each project/phase/to-do, eliminating the internal and project related email;

- Problem solving support - tickets management integrated with all entities.

The HPM solution relies on an ASP.NET web application and takes advantage of Microsoft SharePoint foundation services. The application runs on Microsoft Windows Server on top of Microsoft SharePoint Foundation Server and its data model is implemented in SQL Server RDBM System.

LINQ-TO-SQL is used to enhance the connection between server side C\# programming and database. Several libraries are used in order to integrate the application with native windows functionalities as well as Office tools, which are widely used by knowledge intensive workers. The process manager UI is a totally webbased HTML5 and JavaScript technologies enabled. Several control libraries such as telerik for AJAX.net and HighchartsJS were also employed in order to have a rich and high usable and flexible interface.

During the requirements elicitation process, it was clear that all stakeholders use Microsoft productivity tools in their daily work. Thus, it becomes obvious that a seamlessly integration with Microsoft productivity tools such as windows explorer, outlook and office tools is critically necessary.

Giving this context, is was decided to develop the application on top of .NET and SharePoint foundation frameworks in order to take advantage of its functionalities. Thus, the eApp is designed to run on windows server IIS using Microsoft SQL server and SharePoint Foundation services. Nevertheless, a Windows Communication 
Foundation (WCF) RESTful API was developed in order to have an integration endpoint with any kind of application such as java EE, android, iOS and others. Moreover, MVC Frameworks such as AngularJS can be employed to develop customized GUIs for specific devices like smartphones or tablets.

\section{Validation}

During the process analysis and re-definition, a set of Key Performance Indicators (KPIs) was defined in order to measure the impact of the solution. Thus, for the successful implementation of the solution, the following KPIs were defined:

Overall Report Lead Time (ORLT): Required time necessary to create an overall report in hours is given by the equation 1 .

$$
\text { ORLT }=\frac{\sum(\text { ReportTime }- \text { requestTime })}{\sum \text { reports }}
$$

Overall Report Resources (ORR): Required resources necessary to create an overall report in persons is given by equation 2 .

$$
O R R=\frac{\sum(\text { numberOfResources })}{\sum \text { reports }}
$$

Reaction Time on Critical Status (RTCS): The Reaction Time on Critical Status KPI, measures the mean elapsed time between the problems raise moment till its closing, when they're solved. It's measured in hours and can be calculated using the equation 3 .

$$
\mathrm{RTCS}=\frac{\sum(\text { issueSolvedTime }- \text { issueRaisedTime })}{\sum \text { issues }}
$$

Phase Lead Time (PLT): The Mean time between the start of a project type phase and the effective closing of that phase. This KPI is measured in hours and can be calculated using the equation 4.

$$
P L T=\frac{\sum(\text { EndPhaseTime }- \text { StarPhaseTime })}{\sum \text { phaseInstances }}
$$

Project Schedule Variance (PSV): The variance between the project schedule and its real execution time can be calculated using equation 5 .

$$
P S V=\frac{\sum(\text { RealEndPhaseTime }- \text { RealStarPhaseTime })}{\sum(\text { EstimatedEndPhaseTime }- \text { EstimatedStarPhaseTime })}
$$


The solution was evaluated in real engineering environment inside the company, by the stamping planning team, which involves eight planners and a manager for each project. During this evaluation phase, a specific project has been used to test and verify the functionalities implemented. Each stakeholder has a specific credential to access, test and validate the functionalities, giving feedback for continuous improvement.

It was possible to measure and calculate the KPIs previously defined and compare the results, lead to the savings that are presented in the following table.

Table 1. KPI values and savings.

\begin{tabular}{lllll}
\hline KPI Acronym & Units & AS-IS Result & Actual Result & Savings (\%) \\
\hline TACD & minutes & 30 & 5 & $83,0 \%$ \\
ORLT & hours & 3 & 0,5 & $83,0 \%$ \\
ORR & persons & 9 & 2 & $77,8 \%$ \\
RTCS & hours & 20 & 5 & $75,0 \%$ \\
PLT & months & 4 & 3,5 & $12,5 \%$ \\
\hline
\end{tabular}

\section{Conclusions}

As shown by the results of KPIs from previous section, the introduction of the Hybrid Process Manager in the automotive engineering collaborative environment prove to be very effective and efficient, leading to good results in terms of savings.

The introduction of the HPM made possible that managers avoid asking for reports and a lot of time and paperwork is now saved. HPM allowed planners to concentrate in important issues improving the product and avoid non-value added efforts and time on collateral activities. Other advantage is the data collection built in the solution storing historic and experience retrieval modules, allow access to expertize, knowledge and best practices collected from previous projects and available to incorporate in future as a factor of knowledge sustainability.

The comprehensive dashboard allows the real time report of the project status, leading to savings in reporting time and resources.

Due to integrated documentation and information management, team members have now instant access to the right information at the right place and in the right moment, leading to a reduction of time to access critical data.

The communication functionalities and issues tracking, involving the allowed key players to participate more actively and fostering the team spirit.

This solution has proven to be very effective and efficient when applied in hybrid work systems and in particular in the automotive engineering collaborative processes.

In summary, the project has improved the communication, the cooperation and trust the exchanged information, which are key factors for a successful project. In future, with the integration of suppliers, the solution aims to allow a chain reaction and a huge impact on speeding up the product development and time to market allowing also a faster response to external changes. 
Acknowledgments. The research leading to these results has received funding from the European Union Seventh Framework Programme (FP7/2007-2013) under grant agreement $\mathrm{n}^{\circ} 314156$. The authors would like to thank INESC TEC for their support and the Apps4aME Collaborative Project for their inputs and contributions.

\section{References}

1. S. Kemppilä and P. Mettänen, "Innovations in Knowledge Intensive Services," in Innovations in Knowledge Intensive Services, pp. 22-25 (2004)

2. B. Weber, M. Reichert, and S. Rinderle-Ma, "Change Patterns and Change Support Features - Enhancing flexibility in process-aware information systems," Data Knowl. Eng., vol. 66, pp. 438-466 (2008)

3. C. Hill, R. Yates, C. Jones, and S. L. Kogan, "Beyond predictable workflows: Enhancing productivity in artful business processes," IBM Syst. J., vol. 45, no. 4, pp. 663-682 (2006)

4. M. Adams, A. H. M. Hofstede, D. Edmond, and W. M. P. Van Der Aalst, "Implementing Dynamic Flexibility in Workflows using Worklets," (2006)

5. R. Lu, S. Sadiq, and G. Governatori, "On managing business processes variants," Data Knowl. Eng., vol. 68, no. 7, pp. 642-664 (2009)

6. P. Mangan and S. Sadiq, "On Building Workflow Models for Flexible Processes," J. Aust. Comput. Sci. Commun., vol. 24, no. 2, pp. 103-109 (2002)

7. K. D. Swenson, Mastering the Unpredictable: How Adaptative Case Management Will Revolutionize the Way that Knowledge Workers Get Things Done. Tampa, Florida: MeghanKiffer Press, p. 337 (2010)

8. Bider, "State-Oriented Business Process Modeling: Principles, Theory and Practice," Royal Institute of Technology and Stockholm University (2004)

9. W. M. P. Van Der Aalst, M. Adams, A. H. M. Hofstede, and M. Pesic, "Flexibility as a Service," in Database Systems for Advanced Applications Lecture Notes in Computer Science, pp. 319-333 (2009)

10. Faria J., Silva J. and Marques H., "Supporting the Operation of Semi-structured Work Systems", in ENTERprise Information Systems, International Conference, CENTERIS 2010, Viana do Castelo, Portugal, October 20-22, 2010, Proceedings, Part II, pp 416-425 (2010)

11. Faria J. and Nóvoa H., "An Agile BPM System for Knowledge-Based Service Organizations", in Exploring Services Science, 6th International Conference, IESS 2015, Porto, Portugal, February 4-6, 2015, Proceedings, pp 65-79 (2015)

12. S. W. Sadiq, M. E. Orlowska, and W. Sadiq, "Specification and validation of process constraints for flexible workflows," Inf. Syst., vol. 30, no. 5, pp. 349-378 (2005)

13. W. M. P. van der Aalst, M. Weske, and D. Grünbauer, "Case handling: a new paradigm for business process support,” Data Knowl. Eng., vol. 53, no. 2, pp. 129-162 (2005) 